\title{
Low-dimensional modeling of streaks in a wedge flow boundary layer
}

\author{
María Higuera and José M. Vega \\ E.T.S. Ingenieros Aeronáuticos, Universidad Politécnica de Madrid, \\ Plaza Cardenal Cisneros, 3, 28040-Madrid, Spain
}

(Received 30 November 2011; accepted 21 March 2012; published online 4 May 2012)

\begin{abstract}
This paper is concerned with the low dimensional structure of optimal streaks in a wedge flow boundary layer, which have been recently shown to consist of a unique (up to a constant factor) three-dimensional streamwise evolving mode, known as the most unstable streaky mode. Optimal streaks exhibit a still unexplored/unexploited approximate self-similarity (not associated with the boundary layer self-similarity), namely the streamwise velocity re-scaled with their maximum remains almost independent of both the spanwise wavenumber and the streamwise coordinate; the remaining two velocity components instead do not satisfy this property. The approximate self-similar behavior is analyzed here and exploited to further simplify the description of optimal streaks. In particular, it is shown that streaks can be approximately described in terms of the streamwise evolution of the scalar amplitudes of just three one-dimensional modes, providing the wall normal profiles of the streamwise velocity and two combinations of the cross flow velocity components; the scalar amplitudes obey a singular system of three ordinary differential equations (involving only two degrees of freedom), which approximates well the streamwise evolution of the general streaks. (c) 2012 American Institute of Physics. [http://dx.doi.org/10.1063/1.4711371]
\end{abstract}

\section{INTRODUCTION}

The study of transitional boundary layers has attracted the attention of fluid dynamicists and engineers in the last eighty years due to both its basic interest and its crucial role in numerous engineering applications. Tollmien-Schlichting waves have been identified as the main responsible for the laminar-turbulent transition when the levels of external perturbations are low. However, moderate levels of free-stream turbulence or surface roughness of the wall are able to induce 3D perturbations undergoing non-negligible spanwise variations, alternating low and high momentum within the boundary layer, which amplify and trigger the transition to turbulence. ${ }^{1,2}$ These perturbations are low frequency, streamwise elongated, and the associated streamwise velocity is much larger than (namely, $O(\sqrt{\mathrm{Re}})$ times) the cross flow velocity components. Due to their structure they are called streaks. Streaky perturbations in shear layers are characterized by an initial algebraic growth followed by exponential decay. This behaviour is known as transient growth. ${ }^{3,4}$ Linear physical mechanisms for transient growth in the Blasius boundary layer ${ }^{5,6}$ were extended to the Falkner-Skan boundary layer by Tumin, ${ }^{7}$ who derived an eigenvalue problem describing the velocity components of the streaks, near the leading edge. He computed the largest eigenvalue of this problem and found that the unbounded growth is suppressed as the wedge angle exceeds a threshold value, which is slightly larger than $\pi / 3$. Increasing the wedge angle produces streamwise favorable pressure gradient in the boundary layer, which stabilizes the streaks.

Since transient growth may happen when all modes converge to zero for large time, the simplest results from standard stability theory are not directly applicable. Instead, Butler and Farrell ${ }^{8}$ set the initial valued problem within an optimization context and determined the initial perturbation that generates the maximum growth of the perturbed kinetic energy, at a given later time. Based on the concept of optimal perturbation but considering the spatial growth (instead of temporal), Andersson et al. ${ }^{9}$ and Luchini, ${ }^{10}$ calculated the streamwise optimal perturbation in the case of the 
Blasius boundary layer, using a direct-adjoint technique. This method was also used by Tumin and Ashpis ${ }^{11}$ to compute the optimal streaks in wedge flow (Falkner-Skan) boundary layer, showing that the unbounded growth is suppressed as the wedge angle exceeds a threshold value, which is slightly larger than $\pi / 3$. An interesting property of streaky perturbations in both the Blasius and wedge flow boundary layers is that the spatially evolving streamwise velocity component distribution along the wall normal direction is almost independent of both the streamwise coordinate and the spanwise wavenumber. This approximate self-similar behavior (unexpected, since it is not related to the exact boundary layer self-similarity of the governing equations) has been observed both experimentally ${ }^{12}$ and numerically, ${ }^{9-11}$ and opens up the possibility for obtaining low dimensional descriptions.

The unified framework of internal streaks (driven by localized rugosity/obstacles inside the boundary layer, near the leading edge), where the algebraic growth near the leading edge, the final viscous decay, and the self-similarity of the boundary layer equations are combined, has been recently provided by the authors of the present paper in both the Blasius ${ }^{13}$ and the wedge flow ${ }^{14}$ boundary layers. The main conclusion was that the optimal perturbation may be regarded as just one (up to a constant factor) most unstable streaky mode (MUSM), in the sense that it can be defined in terms of just one streamwise-evolving solution of the linearized equations, with well defined initial conditions near the leading edge. Such MUSM is a joint three-dimensional mode that involves all velocity components.

These results were a step further in identifying the low dimensional nature of optimal streaky perturbations, but (i) the calculation of the MUSM still requires to integrate the full set of linearized boundary layer equations and (ii) the remaining velocity components do not show any approximate self-similarity (see Fig. 5 below). Thus, exploiting the approximate self-similarity to obtain a low dimensional description is not trivial. Instead, it requires to reformulate the problem and to uncover some additional approximate similarity properties of the equations. A first step in this direction results from the observation ${ }^{13,14}$ that the wall normal profile of a certain linear combination of the cross flow velocity components also remains proportional to itself along the streamwise coordinate. It is important to realize that to have an unique three-dimensional mode in the streamwise/cross flow phase space does not imply that its projection in the cross flow subspace is one-dimensional, neither low dimensional. In fact, the velocity field associated to the MUSM is defined as $(u, v, w)=(U(x, y, z), V(x, y, z), W(x, y, z))$ and its projection in the cross flow subspace, $(u, v, w)=\sum A_{j}(x)\left(U_{j}(y, z), V_{j}(y, z), W_{j}(y, z)\right)$, is infinite dimensional.

Against this background, the object of this paper is two-fold. On one hand, the above mentioned approximate self-similarity properties of the boundary layer equations will be completed, as the basic ingredients to low dimensional reduction. On the other hand, a two degrees of freedom model will be derived that provides results that compare well with their exact counterparts. In doing that, some basic principles of low dimensional modeling will be revisited and applied that are of interest in related problems.

Low-dimensional models (also called reduced order models and surrogate models, depending on the context) are the object of current activity in various fields due to their ability to produce fairly precise results at a reasonable computational cost. Such models are of great interest in industrial environments, where reducing both product development cost and time is becoming a crucial issue for competitiveness in the current global market scenario. The fundamental idea behind the deduction of these models is the fact that, although a detailed numerical description of realistic flows requires a large number of mesh points, the approximate fluid behavior involves a moderate amount of modes, and only a few of these are relevant to the dynamics with the rest slaved to these dominant modes. A general path to derive reduced order models consists in projecting the governing equations onto the low dimensional manifold spanned by some proper orthogonal decomposition (POD) modes, obtained from a set of numerically calculated/experimentally measured snapshots, which should be representative of the dynamics that is being described. The use of this technique in turbulence was first introduced by Lumley. ${ }^{15}$ Low dimensional models for turbulent/transitional near-wall and channel flows have been derived using POD-modes and introducing some drastic approximations to the Navier-Stokes equations. ${ }^{16-20}$ Also, more recently Hellström et al. ${ }^{21}$ have shown that the structures of the very-large-scale motions in turbulent pipe flow can be reconstructed using a small number of POD-modes. Interesting and flexible as they are, such general methods of deriving low 
dimensional models emphasize reduction of computational time and RAM memory requirements, more than understanding the underlying physics. In particular, no explicit advantage is taken in cases in which low dimensionality manifests itself in some physically meaningful flow variables, as is the case with approximately self-similar streaks. Taking into account explicitly the approximate self-similarity requires a more ad hoc treatment, but allows a more drastic and physically meaningful reduction of the relevant number of degrees of freedom.

The remaining of the paper is organized as follows. The linearized problem yielding streaky perturbations is formulated and re-scaled in Sec. II. Low dimensional modeling is considered in Sec. III, where the main ingredients of the above mentioned approximate self-similarity are first identified in Subsection III A, and then used to derive the above mentioned two degrees of freedom model in Subsection III B, where comparison with the exact linearized boundary layer equations is also made. The paper ends with some concluding remarks, in Sec. IV.

\section{FORMULATION AND SCALING}

The starting point is the incompressible continuity and Navier-Stokes equations in the usual boundary layer scaling/approximation, assuming a spanwise oscillation with a period comparable to the boundary layer thickness, $\delta^{*}=L^{*} / \sqrt{\mathrm{Re}}$, where $L^{*}$ is the distance to the leading edge and $\operatorname{Re}=u^{*} L^{*} / v \gg 1$ is the Reynolds number based on the free stream velocity $u^{*}$. Nondimensionalization is made in terms of the usual units: $L^{*}$ for the stream wise coordinate $x$, $\delta^{*}$ for the wall normal and spanwise coordinates, $u^{*}$ for the streamwise velocity, $u^{*} / \sqrt{\operatorname{Re}}$ for the wall normal and spanwise velocity components, and $\rho^{*}\left(u^{*}\right)^{2}$ for the pressure. Linear perturbations of the basic, almost parallel, 2D steady state are decomposed in normal modes as $(u, v, w, p)=\left(u_{b}, v_{b}, 0, p_{b}\right)+(U, V, \mathrm{i} W, P / \operatorname{Re}) \mathrm{e}^{\mathrm{i} \alpha z}+\ldots$, to obtain

$$
\begin{aligned}
& \partial_{x} U+\partial_{y} V-\alpha W=0, \\
& \partial_{x}\left(u_{b} U\right)+v_{b} \partial_{y} U+V \partial_{y} u_{b}=\partial_{y y} U-\alpha^{2} U, \\
& u_{b} \partial_{x} V+U \partial_{x} v_{b}+\partial_{y}\left(v_{b} V\right)=-\partial_{y} P+\partial_{y y} V-\alpha^{2} V, \\
& u_{b} \partial_{x} W+v_{b} \partial_{y} W=-\alpha P+\partial_{y y} W-\alpha^{2} W,
\end{aligned}
$$

where $\partial_{x}, \partial_{y}, \cdots$ denote hereafter partial derivatives. The boundary conditions are

$$
U=V=W=0 \quad \text { at } y=0 \text { and } \infty, \quad P=0 \text { as } y \rightarrow \infty .
$$

The basic flow is the Falkner-Skan ${ }^{22}$ self-similar solution

$$
u_{b}(x, \zeta)=x^{\frac{\beta}{2-\beta}} F^{\prime}(\zeta), \quad v_{b}(x, \zeta)=-\frac{F(\zeta)+(\beta-1) \zeta F^{\prime}(\zeta)}{(2-\beta) g(x)},
$$

where the rescaled wall normal coordinate $\zeta$ and the streamfunction $F$ is given by

$$
\begin{aligned}
& \zeta=y / g(x), \quad \text { with } \quad g(x)=x^{\frac{1-\beta}{2-\beta}}, \\
& F^{\prime \prime \prime}+\left[F F^{\prime \prime}+\beta\left(1-F^{\prime 2}\right)\right] /(2-\beta)=0 \quad \text { in } \quad 0<\zeta<\infty, \\
& F(0)=F^{\prime}(0)=0, \quad F^{\prime}(\infty)=1 .
\end{aligned}
$$

The wedge flow solution (Fig. 1) requires that $0 \leq \beta<2$; negative values of $\beta$ are unphysical in wedge flow, unless an additional pressure gradient is imposed. Using the boundary layer selfsimilarity, the spanwise wavenumber $\alpha$ can be removed from the formulation, re-scaling variables 
(a)

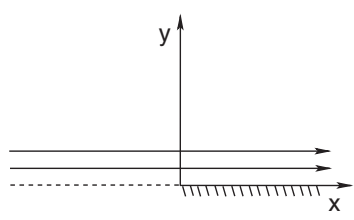

(c)

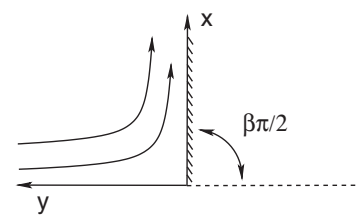

(b)

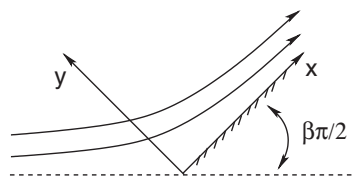

(d)

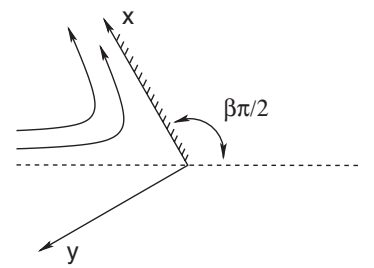

FIG. 1. The symmetric flow past a wedge with angle $\pi \beta$, in the cases (a) $\beta=0$ (Blasius flow), (b) $0<\beta<1$, (c) $\beta=1$ (stagnation flow), and (d) $1<\beta<2$.

as

$$
\left(\hat{x}, \hat{y}, \hat{u}_{b}, \hat{v}_{b}\right)=\left(\alpha^{\frac{2-\beta}{1-\beta}} x, \alpha y, \alpha^{\frac{\beta}{1-\beta}} u_{b}, \frac{v_{b}}{\alpha}\right), \quad(\hat{U}, \hat{V}, \hat{W}, \hat{P})=\frac{\alpha^{\frac{1}{1-\beta}} x}{g(x)}(U, V, W, P) .
$$

This rescaling is useless at $\beta=1$ because $\alpha^{1 /(1-\beta)} \equiv 1, \hat{x} \equiv x$, and $g(x) \equiv 1$ in this case, meaning that $\alpha$ cannot be eliminated from the formulation; but the right hand sides of Eqs. (13)-(17) become independent of $x$ in this case, which represents a transition between two qualitatively different behaviors ${ }^{14}$ of the solutions as $x \rightarrow 0$. Thus, the properties of the streaks that are described below will be lost as $\beta$ approaches 1 from below. In any event, this case will be excluded from the analysis below, which will concentrate in those values of $\beta$ that provide transient growth. The asymptotic analysis as $\zeta \rightarrow \infty$ of the resulting linearized boundary layer equations ${ }^{13,14}$ shows that the behavior of streaky perturbations near the free stream is slaved to the solution inside of the boundary layer, and such that

$$
(\hat{U}, \hat{V}, \hat{W}, \hat{P})=\left(0, \hat{V}_{\infty}(\hat{x}), \hat{W}_{\infty}(\hat{x}), \hat{P}_{\infty}(\hat{x})\right) \mathrm{e}^{-g(\hat{x})\left(\zeta-a_{\beta}\right)}+\mathrm{UEST},
$$

where $\hat{V}_{\infty}, \hat{W}_{\infty}$, and $\hat{P}_{\infty}$ are such that $g(\hat{x})^{2} \hat{V}_{\infty}^{\prime}=\hat{V}_{\infty} \frac{(1+\beta)-\beta\left(\zeta-a_{\beta}\right) g(\hat{x})}{2-\beta}+g(\hat{x}) \hat{P}_{\infty}$ and $\hat{V}_{\infty}+\hat{W}_{\infty}=0$ and UEST stands for uniformly, exponentially small terms as $\zeta \rightarrow \infty$, which converge to zero as $\mathrm{e}^{-\left(\zeta-a_{\beta}\right)^{2} / 2}$ times a power of $\zeta$. Thus, $\hat{V}, \hat{W}$, and $\hat{P}$ converge quite slowly near the leading edge, when $\hat{x}$ is small, and the leading order term in Eq. (11) captures quite well this behavior. This result clearly suggests the convenience of replacing $\hat{W}$ by the variable

$$
\hat{H}=\hat{W}+\hat{V},
$$

which behaves as $\exp \left[-\left(\zeta-a_{\beta}\right)^{2} / 2\right]$ at large $\zeta$. Using Eq. (12) and scaling Eq. (10), the linearized boundary layer equations (1)-(5) take the form

$$
\begin{aligned}
& \hat{x} \partial_{\hat{x}} \hat{U}=\frac{1-\beta}{2-\beta} \zeta \partial_{\zeta} \hat{U}-\partial_{\zeta} \hat{V}-g(\hat{x}) \hat{V}+g(\hat{x}) \hat{H} \\
& F^{\prime} \hat{x} \partial_{\hat{x}} \hat{U}=\partial_{\zeta \zeta} \hat{U}+\frac{F}{2-\beta} \partial_{\zeta} \hat{U}-\frac{\beta F^{\prime}+(\beta-1) \zeta F^{\prime \prime}+(2-\beta) g(\hat{x})^{2}}{2-\beta} \hat{U}-F^{\prime \prime} \hat{V} \\
& F^{\prime} \hat{x} \partial_{\hat{x}} \hat{V}=\partial_{\zeta \zeta} \hat{V}+\frac{F}{2-\beta} \partial_{\zeta} \hat{V}+\frac{(\beta-1)\left[F+(2 \beta-1) \zeta F^{\prime}+(\beta-1) \zeta^{2} F^{\prime \prime}\right]}{(2-\beta)^{2}} \hat{U} \\
& +\frac{(\beta+1) F^{\prime}+(\beta-1) \zeta F^{\prime \prime}-(2-\beta) g(\hat{x})^{2}}{2-\beta} \hat{V}-\partial_{\zeta} \hat{P}
\end{aligned}
$$




$$
\begin{aligned}
& \hat{x} F^{\prime} \partial_{\hat{x}} \hat{H}= \partial_{\zeta \zeta} \hat{H}+\frac{F}{2-\beta} \partial_{\zeta} \hat{H}+\frac{(\beta-1)\left[F+(2 \beta-1) \zeta F^{\prime}+(\beta-1) \zeta^{2} F^{\prime \prime}\right]}{(2-\beta)^{2}} \hat{U} \\
&+\frac{\beta F^{\prime}+(\beta-1) \zeta F^{\prime \prime}}{2-\beta} \hat{V}+\frac{F^{\prime}-(2-\beta) g(\hat{x})^{2}}{2-\beta} \hat{H}-\partial_{\zeta} \hat{P}-g(\hat{x}) \hat{P}, \\
& \hat{U}=\hat{V}=\hat{H}=0 \quad \text { at } \quad \zeta=0 \text { and }, \quad \hat{U}, \hat{H}, \hat{P} \rightarrow 0 \quad \text { as } \quad \zeta \rightarrow \infty .
\end{aligned}
$$

These are the equations that will be considered along the paper and will be referred to as the modified linearized boundary layer (MLBL) equations.

\section{LOW DIMENSIONAL MODELING}

Let us first analyze the approximate self-similar structure of streaks, in Subsection III A, which provides the main ingredients that are essential for the derivation of the above mentioned two-dimensional model, in Subsection III B.

\section{A. Approximate self-similarity}

To begin with, we integrate the system (13)-(17) using a quite precise and numerically inexpensive solver, ${ }^{13,14}$ which allows for integrating from extremely small values of $\hat{x}$. As anticipated above (see also Fig. 4 below), if arbitrary initial conditions are imposed at small $\hat{x}$ (say, $\hat{x}=\hat{x}_{0}<10^{-3}$ ) then, after a transient $\left(\hat{x}_{0}<\hat{x}<10 \hat{x}_{0}\right.$, typically), all solutions of Eqs. (13)-(17) converge to the MUSM, and the wall normal profiles of both $\hat{U}$ and $\hat{H}$ remain almost constant when rescaled with their maxima (see Fig. 2). Now, a look at the continuity equation (13) reveals that because $\hat{U}$ and $\hat{H}$ can be both approximately described using one mode, the term $\zeta \partial_{\zeta} \hat{U} / 2$ is also described by one mode. Thus, the remaining terms in Eq. (13), namely $\partial_{\zeta} \hat{V}+g(\hat{x}) \hat{V}$, must be also approximately described as a linear combination of at most three modes. In fact, Fig. 2 shows that $\partial_{\zeta} \hat{V}+g(\hat{x}) \hat{V}$ is approximately described in terms of only one mode. Thus, we have three quantities depending on the velocity components that can be described in terms of one mode each. There is no obvious quantity involving the pressure that can be described in terms of just one mode, but this is not essential, since pressure can be eliminated from the formulation.

Note also that the approximate self-similarity of the quantities $\hat{U}, \hat{H}$, and $\partial_{\zeta} \hat{V}+g(\hat{x}) \hat{V}$ applies for all $\hat{x}$, in particular as $\hat{x} \ll 1$. Thus, these quantities must coincide with their counterparts in the asymptotic behavior as $\hat{x} \rightarrow 0$, which was first calculated by Tumin. ${ }^{7}$ More recently, this problem was re-interpreted ${ }^{14}$ invoking Eq. (11), which suggests rewriting the flow variables as

$$
(\hat{U}, \hat{V}, \hat{H}, \hat{P}) \sim \hat{x}^{\lambda}(\tilde{U}, \tilde{V}, \tilde{H} / g(\hat{x}), \tilde{P}) e^{-g(\hat{x})\left(\zeta-a_{\beta}\right)} .
$$
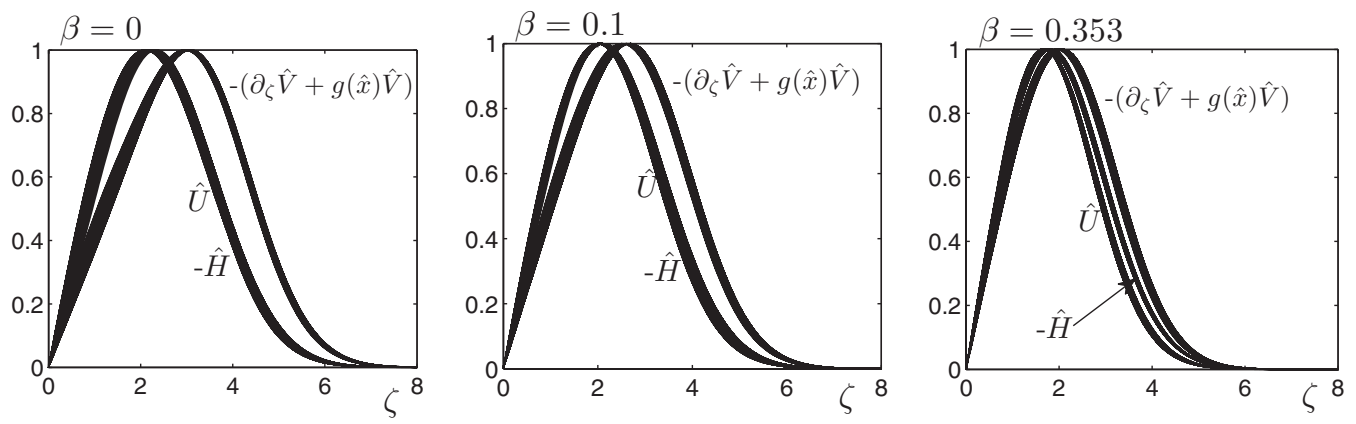

FIG. 2. Wall normal profiles (re-scaled with their maxima) of $\hat{U},-(\hat{V}+g(\hat{x}) \hat{V})$, and $-\hat{H}$ on the MUSM' $10^{-6}$ and 1 for the indicated values of $\beta$. 


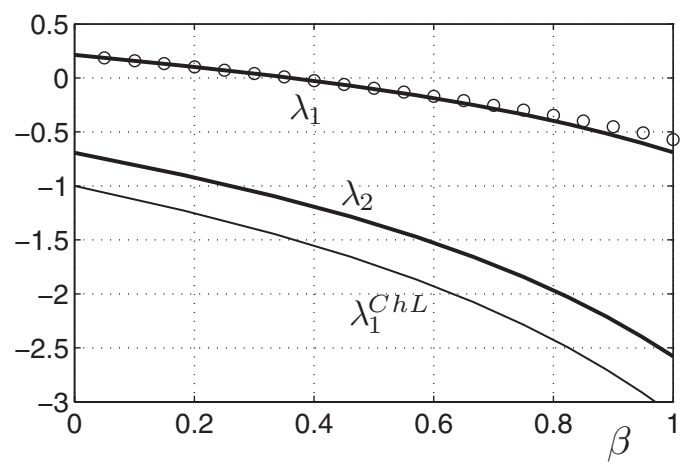

FIG. 3. The two largest Tumin eigenvalues, $\lambda_{1}$ and $\lambda_{2}$ and the largest Chen-Libby eigenvalue, $\lambda_{1}^{\mathrm{ChL}}$ in terms of $\beta$ : as calculated from the exact eigenvalue problems (solid lines) and as provided by the reduced model (plain circles), according to Eq. (43) below.

Substituting Eq. (18) into Eqs. (13)-(17) and retaining only the leading order terms as $\hat{x} \rightarrow 0$ yield

$$
\begin{aligned}
& \lambda \tilde{U}+\frac{(\beta-1) \zeta}{2-\beta} \tilde{U}^{\prime}+\tilde{V}^{\prime}-\tilde{H}=0, \\
& \tilde{U}^{\prime \prime}+\frac{F}{2-\beta} \tilde{U}^{\prime}+\frac{(1-\beta) \zeta F^{\prime \prime}-[(2-\beta) \lambda+\beta] F^{\prime}}{2-\beta} \tilde{U}-F^{\prime \prime} \tilde{V}=0, \\
& \tilde{H}^{\prime \prime}+\frac{F}{2-\beta} \tilde{H}^{\prime}+(1-\lambda) F^{\prime} \tilde{H}=0, \\
& \tilde{U}=\tilde{V}=\tilde{H}=0, \quad \text { at } \zeta=0, \quad \tilde{U}, \tilde{V}^{\prime}, \tilde{H} \rightarrow 0 \text { as } \zeta \rightarrow \infty,
\end{aligned}
$$

whose eigenvalues (the largest two are plotted in Fig. 3) are given by the third equation, which is decoupled. The most dangerous eigenvalue is the first one, which decreases as $\beta$ increases, from $\lambda_{1}$ $=0.2195$ at $\beta=0$ to 0 at $\beta=\beta_{c}=0.362$ and is negative if $\beta>\beta_{c}$; the remaining eigenvalues are all negative. Note that only the positive eigenvalues promote algebraic growth. The eigenfunction components of the first Tumin mode rescaled with their maxima will be denoted hereafter as $\tilde{U}_{1}, \tilde{V}_{1}$, and $\tilde{H}_{1}$, and $\tilde{U}_{1}, \tilde{V}_{1}^{\prime}$, and $\tilde{H}_{1}$ are plot indistinguishable from their counterparts in Fig. 2 . The decay to the MUSM is provided by the second Tumin eigenvalue, $\lambda_{2}$, as expected from Fig. 3 .

Besides the asymptotic behavior (18), the scaling

$$
(\hat{U}, \hat{V}, \hat{H}, \hat{P}) \sim \hat{x}^{\lambda}(\tilde{U}, \tilde{V}, \tilde{H}, \tilde{P}) e^{-g(\hat{x})\left(\zeta-a_{\beta}\right)}
$$

must also be considered, with the exponent $\lambda$ as calculated from an eigenvalue problem, whose twodimensional version were first considered by Chen and Libby. ${ }^{23}$ As can be seen in Fig. 3, the largest Chen Libby eigenvalue, $\lambda_{1}^{\mathrm{ChL}}$, is smaller than the first two of Tumin, meaning that the associated behavior exhibits faster decay and can be ignored.

Summarizing, three quantities have been identified, namely

$$
\hat{U}, \quad \hat{H}, \quad \text { and } \quad \partial_{\zeta} \hat{V}+g(\hat{x}) \hat{V},
$$

which are approximately described by one mode each. This result comes from an approximate boundary layer self-similarity and constitutes the only essential ingredient in the derivation of the low dimensional model that will be obtained in Sec. III B. The elimination of the wavenumber $\alpha$ that has been made using the exact boundary layer self-similarity through the rescaled variables (10) instead is not essential, but only allows for simplifying the analysis. In other words, $\alpha$ is eliminated introducing a scaling factor $\alpha^{1 /(1-\beta)}$ in the velocity components in Eq. (10), which preserves the fact that the quantities (24) can be approximated by one mode each. Therefore, if the factor $\alpha^{1 /(1-\beta)}$ is not included, the counterparts of the three above mentioned quantities are still approximated by 
one mode each. This means that a low dimensional model could also be derived using the original unscaled variables and proceeding as in the Sec. III B.

\section{B. Low dimensional modeling of streaks}

The application of standard low dimensional modeling based on POD would require to first calculate POD modes from a set of representative snapshots (namely, wall normal profiles of the flow variables for representative values of $\hat{x}$ ) obtained from the exact unstable streaky mode, and then projecting the governing equations (13)-(17) onto the manifold spanned by the POD modes. This method may exhibit instability due to higher order modes truncation, which is the object of current research. ${ }^{24-29}$ If instability is avoided, the root mean square (RMS) error of the solution of the resulting model retaining $n$ modes is readily calculated in terms of the POD singular values as $\sqrt{\sigma_{n+1}^{2}+\sigma_{n+2}^{2}+\ldots}$. Now, taking as snapshots (for the case $\beta=0$ ) the $\zeta$-profiles of the original velocity vector, $(\hat{U}, \hat{V}, \hat{W})$ (with each component rescaled with their maxima) at 100 logarithmically equispaced values of $\hat{x}$ in the interval $0.001 \leq \hat{x} \leq 1$, the first six associated singular values are 1.224, $0.393,0.120,0.043,0.015$, and 0.004 , respectively, which means that five modes would be required in a standard POD-based reduced order model to obtain a relative RMS error of the order of 0.01 ; if instead, the vectors $\left(\hat{U}, \partial_{\zeta} \hat{V}+\sqrt{\hat{x}} \hat{V}, \hat{H}\right)$ are considered, then the singular values are $0.772,0.020$, $0.006,0.001$, and $3 \times 10^{-4}$, which means that two modes are enough to obtain the same relative RMS error. This is easily understood recalling our comments above, in Subsection III A and Fig. 2, and is consistent with the two degrees of freedom model that will be obtained below. Therefore, using the following one mode description for these three variables is expected to provide a reasonable low dimensional modeling:

$$
\hat{U}=A_{1}(\hat{x}) \tilde{U}_{1}(\zeta), \quad \partial_{\zeta} \hat{V}+g(\hat{x}) \hat{V}=A_{2}(\hat{x}) \tilde{V}_{1}^{\prime}, \quad \hat{H}=A_{3}(\hat{x}) \tilde{H}_{1}(\zeta)
$$

where $\tilde{U}_{1}, \tilde{V}_{1}^{\prime}$, and $\tilde{H}_{1}$ can be defined in either of the following ways:

- The POD modes obtained above applying POD to the snapshots $\left(\hat{U}, \partial_{\zeta} \hat{V}+\sqrt{\hat{x}} \hat{V}, \hat{H}\right)$. But using these requires to calculate the snapshots for each value of $\beta$. This must be done using a numerical solver for the MLBL equations (13)-(17).

- Figure 2 shows that each of the quantities (24) are described by just one mode already for small values of $\hat{x}$, and the essence of POD implies that the modes that approximate the quantities (24) must approximately coincide with the above mentioned POD modes. On the other hand, Eq. (23) implies that (recall that $g(\hat{x}) \rightarrow 0$ as $\hat{x} \rightarrow 0$ )

$$
\left(\hat{U}, \partial_{\zeta} \hat{V}+\sqrt{\hat{x}} \hat{V}, \hat{H}\right) \simeq \hat{x}^{\lambda}\left(\tilde{U}, \tilde{V}^{\prime}, \tilde{H}\right),
$$

where $\tilde{U}, \tilde{V}$, and $\tilde{H}$ are the components of the first Tumin mode, which is the dominant one as $\hat{x} \rightarrow 0$. Note that the calculation of the first Tumin mode only requires solving a one-dimensional eigenvalue problem.

The second alternative is more computationally efficient and, furthermore, emphasizes the property that the whole streamwise evolution of the relevant streaks are dominated by the behavior at the leading edge. Thus the modes $\tilde{U}_{1}, \tilde{V}_{1}$, and $\tilde{H}_{1}$ appearing in the description (25) will be taken hereafter as the components of the first Tumin mode. The second expression in Eq. (25) is readily integrated, to obtain

$$
\hat{V}(\hat{x}, \zeta)=A_{2}(\hat{x}) K(\hat{x}, \zeta) \quad \text { where } K(\hat{x}, \zeta)=\mathrm{e}^{-g(\hat{x}) \zeta} \int_{0}^{\zeta} \mathrm{e}^{g(\hat{x}) \eta} \tilde{V}_{1}^{\prime}(\eta) d \eta
$$

Let us now obtain a reduced model that consists in three ODEs for the streamwise evolution of the amplitudes $A_{1}, A_{2}$, and $A_{3}$. To this end, we first eliminate the pressure from Eqs. (15) and (16), 
which leads to

$$
\begin{aligned}
& \hat{x} \partial_{\hat{x}}\left[F^{\prime} \Omega+F^{\prime \prime}(\hat{V}-H)\right]=\partial_{\zeta \zeta} \Omega+\frac{F}{(2-\beta)} \partial_{\zeta} \Omega+\frac{2 F^{\prime}-(2-\beta) g(\hat{x})^{2}}{(2-\beta)} \Omega- \\
& \quad \frac{(\beta-1)\left(F-(2 \beta-1) \zeta F^{\prime}-(\beta-1) \zeta^{2} F^{\prime \prime}\right)}{(2-\beta)^{2}} g(\hat{x}) \hat{U}+\frac{F^{\prime \prime}-g(\hat{x}) \zeta F^{\prime \prime}}{(2-\beta)} \hat{V}-\frac{F^{\prime \prime}}{(2-\beta)} \hat{H},
\end{aligned}
$$

in terms of the streamwise vorticity

$$
\Omega=\partial_{\zeta} \hat{H}-\partial_{\zeta} \hat{V}-g(\hat{x}) \hat{V} .
$$

Substituting Eqs. (25)-(27) into Eqs. (13)-(14) and (28), multiplying the resulting equations by $\tilde{V}_{1}^{\prime}$, $\tilde{U}_{1}$, and $\tilde{H}_{1}^{\prime}$, respectively, and integrating in $0<\zeta<\infty$, yields

$$
\begin{gathered}
\hat{x} A_{1}^{\prime}=-\frac{(1-\beta) \gamma_{1}}{2(2-\beta)} A_{1}+\gamma_{2} A_{2}-\gamma_{3} g(\hat{x}) A_{3}, \\
\hat{x} A_{1}^{\prime}=-\gamma_{4} A_{1}+\Psi_{2} A_{2}, \\
\hat{x}\left(\Psi_{1} A_{2}+A_{3}\right)^{\prime}=-\gamma_{5} g(\hat{x}) A_{1}-\Psi_{3} A_{2}-\gamma_{6} A_{3},
\end{gathered}
$$

which will be called below the reduced model. Note that Eqs. (30), (31), and (32) are precisely the one-mode counterparts of the continuity, streamwise momentum, and streamwise vorticity equations, (13), (14), and (28), respectively. In particular:

- The three terms appearing in Eq. (30) exactly coincide with their counterparts in Eq. (13) after grouping $\partial_{\zeta} \tilde{V}+g(\hat{x}) \tilde{V}$.

- The first term in the right hand side of Eq. (31) accounts for two contributions associated with the two terms that appear in the expression of $\gamma_{4}$, see Eq. (33) below. The first contribution is independent of $\hat{x}$ and results from the leading order effect of the leading edge singularity on the evolution of the streamwise velocity. The second contribution is proportional to $g(\hat{x})^{2}$ and accounts for viscous dissipation. Note that both contributions to $\gamma_{4}$ are positive, which means that they promote streamwise decay in the streamwise velocity component.

- The second (off-diagonal) term in the right hand side of Eq. (31) accounts for the coupling effect of the cross-flow velocity components on the streamwise momentum equation, and is precisely the term that produces the lift up effect in the streaks. In other words, this term promotes the transient growth of the streamwise velocity component that compensates the decay discussed in the last item.

- The various terms in the right hand side of Eq. (32) account for the effects of the three velocity components on the streamwise evolution of the streamwise vorticity.

The coefficients in Eqs. (30)-(32) are calculated as re-scaled (to set to one some of the coefficients) integrals in $0<\zeta<\infty$ of various functions of $\hat{x}$ and $\zeta$, which depend on the latter variable through $\tilde{V}_{1}^{\prime}, \tilde{U}_{1}, \tilde{H}_{1}^{\prime}$ and their derivatives (up to third order); second and third order derivatives can be eliminated using Eqs. (19)-(21). The explicit expressions of these coefficients are given in Eqs. (A1)-(A6) in the Appendix. When integrating Eqs. (30)-(32), the coefficients could be computed at each $\hat{x}$-step performing the integrals appearing in Eqs. (A1)-(A6), but computational efficiency is greatly improved using the following approximations, which are readily obtained

$$
\begin{aligned}
& \gamma_{1}=1, \quad \gamma_{2}=0.98+0.15 \beta, \quad \gamma_{3}=1, \quad \gamma_{4}=(0.47+1.83 \beta)+(1.46-0.38 \beta) g(\hat{x})^{2}, \\
& \gamma_{5}=0.37-2.35 \beta+2.86 \beta^{2}, \quad \gamma_{6}=(0.28+0.28 \beta)+(1.57-0.56 \beta) g(\hat{x})^{2}, \\
& \Psi_{1}=1.19-3.04 \beta+3.01 \beta^{2}-(0.28-0.49 \beta) g(\hat{x})+(0.14-0.24 \beta) g(\hat{x})^{2},
\end{aligned}
$$




$$
\begin{aligned}
& \Psi_{2}=0.35-0.13 \beta-(0.29-0.25 \beta) g(\hat{x})+(0.11-0.13 \beta) g(\hat{x})^{2} \\
& \Psi_{3}=0.05+0.26 \beta-0.53 \beta^{2}+\left(0.52-1.62 \beta+1.74 \beta^{2}\right) g(\hat{x})+\left(0.45-1.61 \beta+1.89 \beta^{2}\right) g(\hat{x})^{2} .
\end{aligned}
$$

Even though Eqs. (30)-(32) are three ODEs, they only involve two degrees of freedom, as seen by eliminating $A_{1}^{\prime}$ from Eqs. (30) to (31), which yields

$$
\left.\left[(1-\beta) /(2-\beta)-2 \gamma_{4}\right]\right) A_{1} / 2+\left(\Psi_{2}-\gamma_{2}\right) A_{2}+g(\hat{x}) A_{3}=0 .
$$

This equation can be regarded as a compatibility condition that must be satisfied by initial conditions. A further substitution of Eq. (38) into Eq. (30) would allow for eliminating either $A_{2}$ or $A_{3}$, to obtain a system of two ODEs, which means that the reduced model (30)-(32) is dynamically two-dimensional. But such reduction is of conceptual interest only. The results below are obtained integrating Eqs. (30)-(32).

As a first test to check this reduced model, we consider the relevant behavior as $\hat{x} \rightarrow 0$,

$$
\left(A_{1}, A_{2}, A_{3}\right)=\hat{x}^{\lambda}\left(A_{1}^{0}, A_{2}^{0}, A_{3}^{0} / g(\hat{x})\right),
$$

which is the counterpart of Eq. (18). Substituting these into Eqs. (30)-(32) and setting $\hat{x}=0$ in the resulting equations lead to the following eigenvalue problem:

$$
\begin{aligned}
& \lambda A_{1}^{0}=-[(1-\beta) /(2-\beta)] A_{1}^{0} / 2+\gamma_{2} A_{2}^{0}-A_{3}^{0}, \\
& \lambda A_{1}^{0}=-\gamma_{4} A_{1}^{0}+\Psi_{2}^{0} A_{2}^{0}, \\
& \lambda A_{3}^{0}=\left[(1-\beta) /(2-\beta)-\gamma_{6}\right] A_{3}^{0},
\end{aligned}
$$

where the last equation is decoupled and yields the following approximation of the first Tumin eigenvalue,

$$
\lambda_{1}=-\gamma_{6}+(1-\beta) /(2-\beta)
$$

which compares quite well with its exact counterpart (see Fig. 3).

The counterpart of the MUSM is obtained integrating Eqs. (30)-(32), taking as initial condition that in the first expression in Eq. (39), with $\lambda=\lambda_{1}$ and the eigenvector $\left(A_{1}^{0}, A_{2}^{0}, A_{3}^{0}\right)$ calculated from Eqs. (40) to (42). Once $A_{1}(\hat{x}), A_{2}(\hat{x})$, and $A_{3}(\hat{x})$ have been calculated, the profiles of the three velocity components are reconstructed using Eqs. (12), (25), and (27). The resulting maximum values along the wall normal direction of $|\hat{U}|,|\hat{V}|$, and $|\hat{W}|$ for the approximated MUSM and the associated rescaled wall normal profiles of $\hat{V}$ and $\hat{W}$ are shown with thick dashed lines in Figs. 4 and 5. For comparison, their exact counterparts calculated from the exact MLBL equations are also plotted with thick solid lines in both figures. Note that a scaling factor (common to all velocity components) is free in both the exact and approximate solutions, plotted in Fig. 4; these scaling factors have been adjusted to make both families of curves close to each other. Note that the exact and approximate solutions remain close to each other in the whole range $10^{-6}<\hat{x}<1$. Solutions of the reduced model with other initial conditions (not associated with the leading order behavior as $\hat{x} \rightarrow 0$ ) are also given with thin dashed lines in Fig. 4. As can be seen, after a transient all solutions converge to the unstable streaky mode, as happens with the exact solutions. ${ }^{14}$ Figure 5 shows that the reduced model produces quite well reconstruction of the wall normal profiles of $\hat{V}$ and $\hat{W} ; \hat{U}$ is not plotted because the approximation is necessarily good according to the approximate self-similarity properties discussed in Subsection III A and the description of $\hat{U}$ in the reduced model, see Eq. (25).

Let us now check the ability of the reduced model to reconstruct optimal perturbations, which are defined as those maximizing the perturbed kinetic energy gain between an initial section, $x=x_{\mathrm{in}}$, 

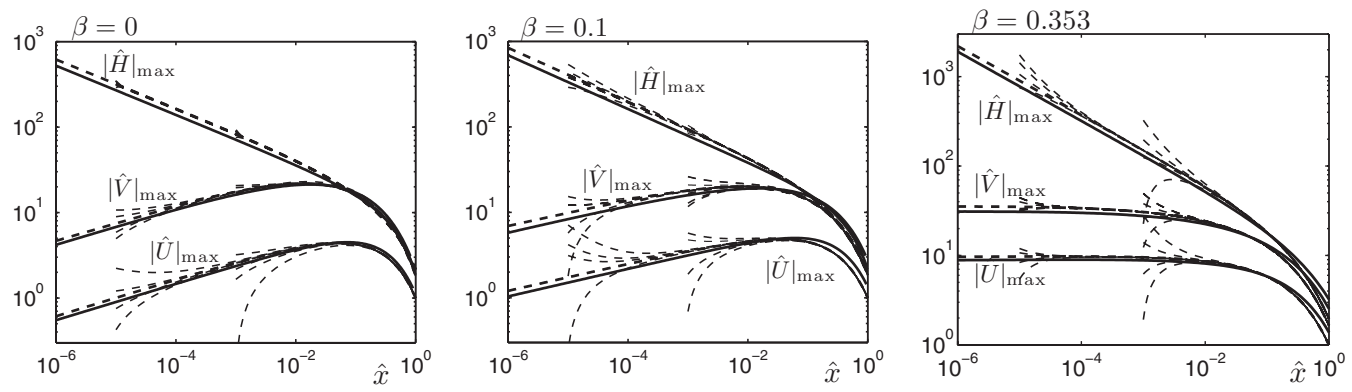

FIG. 4. Maxima (in $0<\zeta<\infty$ ) of $|\hat{U}|,|\hat{V}|$, and $|\hat{W}|$ vs. $\hat{x}$ along the MUSM as calculated from the reduced model (thick dashed lines) and the exact MLBL equations (solid lines), and counterparts calculated with the reduced model using random initial conditions (thin dashed lines).

and $x=1$, for varying spanwise wavenumber $\alpha$ and varying initial conditions. Invoking the rescaling introduced in Sec. II, the maximum gain is approximated as

$$
G_{\max }(\alpha)=\max \frac{\int_{0}^{\infty}\left[U^{2}\right]_{x=1} d y}{\int_{0}^{\infty}\left[\operatorname{Re} U^{2}+\frac{g(x)}{x^{2}}\left(V^{2}+W^{2}\right)\right]_{x=x_{\mathrm{in}}} d y}, \quad \operatorname{Re} \gg 1 .
$$

In order to calculate this gain with the reduced model, we must take into account Eq. (10), which relates the original variables used in Eq. (44) with the re-scaled variables in the MLBL equations. Also, since the reduced model (30)-(32) exhibits two degrees of freedom, a generic solution with initial conditions (at $\left.x_{\mathrm{in}}\right)\left(A_{1}, A_{3}\right)=\left(A_{1}^{0}, A_{3}^{0}\right)$ can be written as a linear combination of those fundamental solutions with initial conditions $\left(A_{1}^{0}, A_{3}^{0}\right)=(1,0)$ and $(0,1)$. Using these, the gain (44) is re-written as

$$
G_{\max }(\alpha)=\max _{\boldsymbol{q}} \frac{\boldsymbol{q}^{T} E^{\mathrm{out}} \boldsymbol{q}}{\boldsymbol{q}^{T} E^{\text {in }} \boldsymbol{q}}
$$
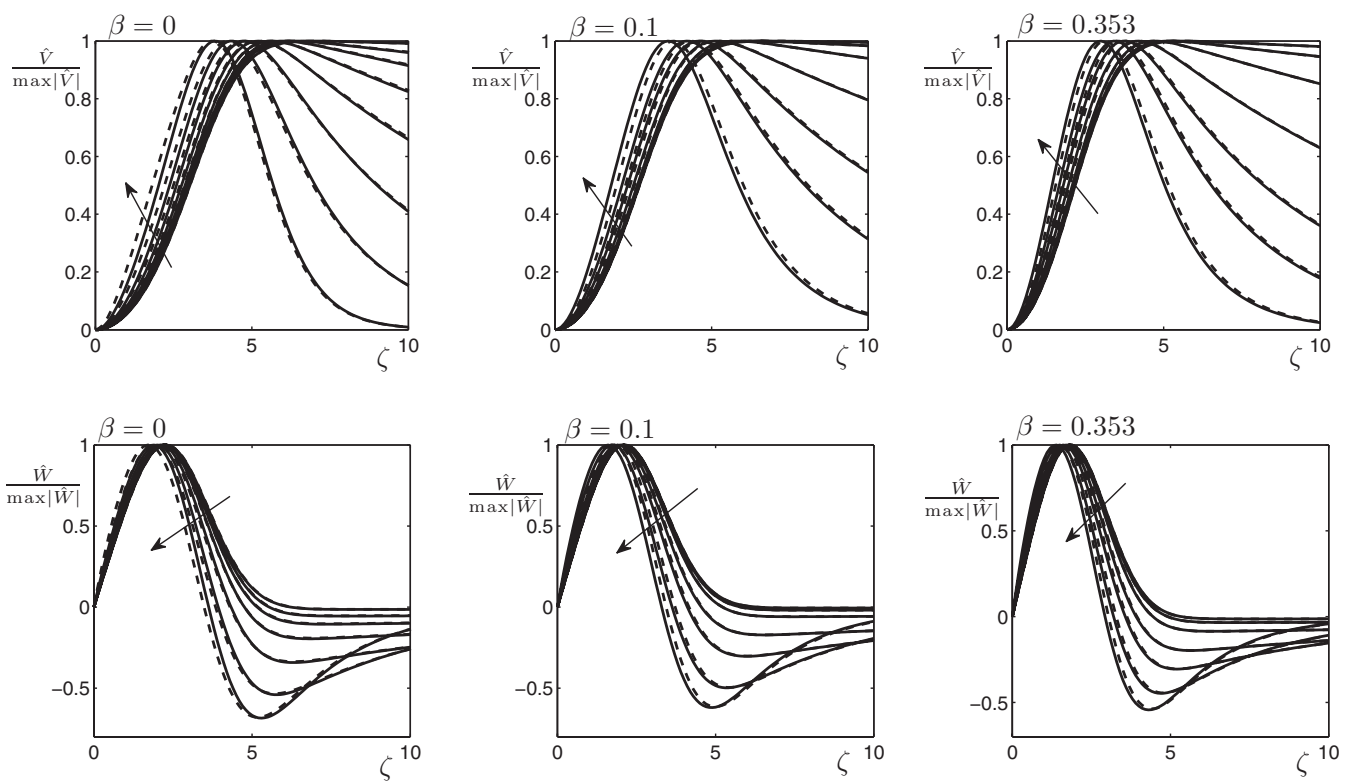

FIG. 5. Cross flow velocity profiles on the MUSM, re-scaled with their maxima, as calculated from the exact MLBL equations (solid lines) and from the reduced model (dashed lines) at $\hat{x}=10^{-6}, 10^{-5}, 10^{-4}, 10^{-3}, 10^{-2}, 10^{-1}$ and 1 . Arrows indicate increasing values of $\hat{x}$. 
where $\boldsymbol{q}^{T}=\left(A_{1}^{0}, A_{3}^{0}\right)$ and (for $\left.i, j=1,2\right)$

$$
\begin{aligned}
E_{i j}^{\mathrm{in}} & =x_{\mathrm{in}}^{-(1+\beta) /(2-\beta)} \int_{0}^{\infty}\left[x_{\mathrm{in}}^{2 /(2-\beta)} \operatorname{Re} \hat{U}_{i} \hat{U}_{j}+\hat{V}_{i} \hat{V}_{j}+\hat{W}_{i} \hat{W}_{j}\right]_{\hat{x}=\alpha^{(2-\beta) /(1-\beta)} x_{\mathrm{in}}} d \zeta, \\
E_{\mathrm{ij}}^{\mathrm{out}} & =\int_{0}^{\infty}\left[\hat{U}_{i} \hat{U}_{j}\right]_{\hat{x}=\alpha^{(2-\beta) /(1-\beta)}} d \zeta .
\end{aligned}
$$

Here, for $i=1$ and $2, \hat{U}_{i}, \hat{V}_{i}$, and $\hat{W}_{i}$ are the velocity profiles reconstructed (using Eqs. (10), (12), (25), and (27)) from the two above mentioned fundamental solutions of the reduced model. For each value of $\alpha, G_{\max }(\alpha)$ is the maximum eigenvalue of $E^{\text {out }} \boldsymbol{q}=\lambda E^{\text {in }} \boldsymbol{q}$, and the maximizers of Eq. (45) are the associated eigenvectors. The maximum of the gain (47) turns out to be independent of the Reynolds number (within plot accuracy) whenever $x_{\mathrm{in}} \operatorname{Re}>0.01$. This is because, according to Eq. (46), the dependence on Re is concentrated in $E_{11}^{\text {in }}$, which is of the form $E_{11}^{\mathrm{in}}=\operatorname{Re} x_{\mathrm{in}}^{(1-\beta) /(2-\beta)} E_{11,0}^{\mathrm{in}}+E_{11,1}^{\mathrm{in}} / x_{\mathrm{in}}^{(1+\beta) /(2-\beta)}$, while the remaining components of $E^{\mathrm{in}}$ and all components of $E^{\text {out }}$ are independent of Re. This means that as $\operatorname{Re} \rightarrow \infty$ the maximum of the gain (45) simplifies to

$$
G_{\max }(\alpha)=E_{22}^{\text {out }} / E_{22}^{\text {in }},
$$

which is also obtained setting $A_{1}^{0}=0$ (namely, setting $U=0$ at $x=x_{\text {in }}$, see Eq. (25)) in the right hand side of Eq. (44). This approximation is illustrated with dashed lines in Fig. 6, left for two values of the initial stage, $x_{\text {in }}=10^{-5}$ and $x_{\text {in }}=0.25$; the exact counterparts ${ }^{13,14}$ are also plotted with solid lines. Note that the approximation is fairly good and that, as already noticed from calculations with the exact MLBL equations, ${ }^{13,14}$ dependence on the initial position $x_{\text {in }}$ is rather weak. In particular, for $\beta=0.353$ (a case considered by Tumin, ${ }^{11}$ whose results are plotted with plain circles), the maxima of the gain curves are attained at

$$
\alpha=0.545 \text { and } 0.553 \text { for } x_{\text {in }}=10^{-5} \text { and } 0.25 \text {, respectively, }
$$

which compare quite well with their exact counterparts, ${ }^{11,14} \alpha \simeq 0.56$ and 0.57 , respectively.
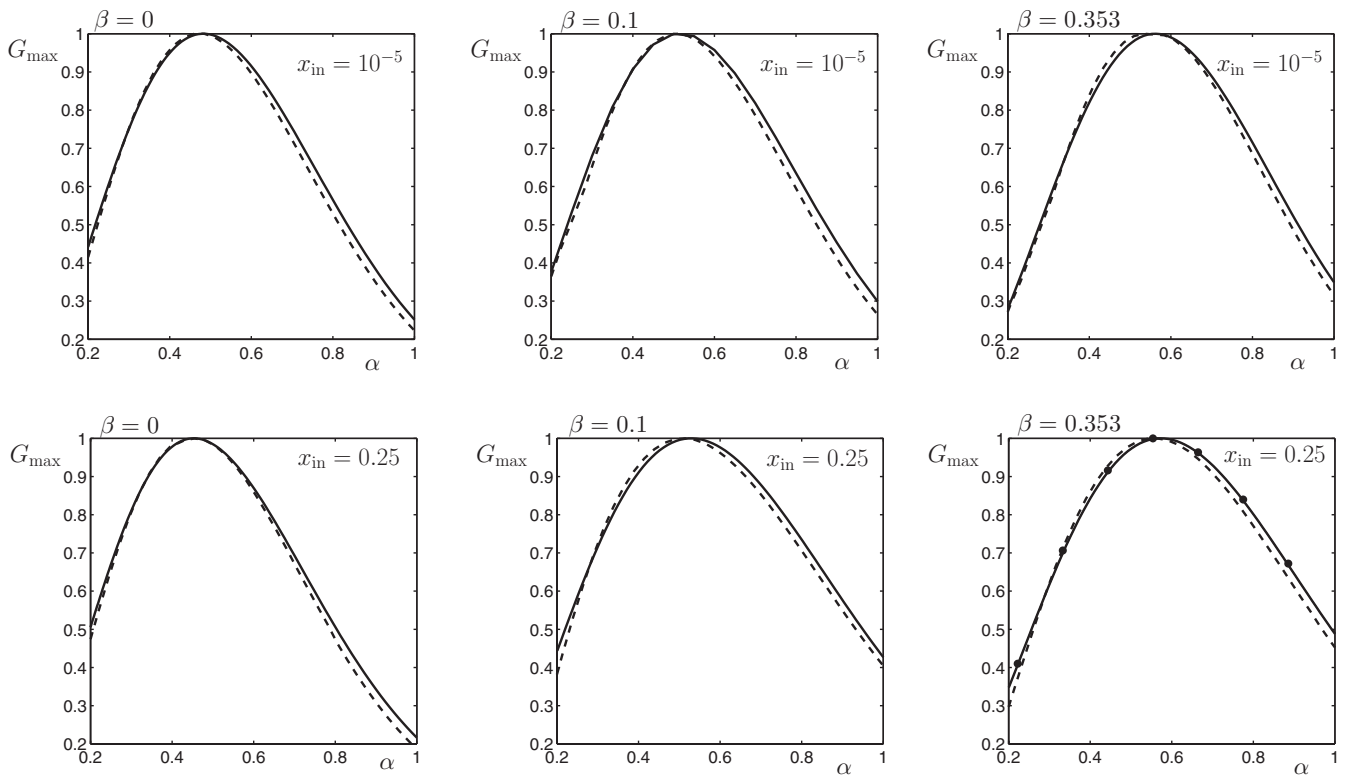

FIG. 6. Left: maximum of "perturbed energy gain" (44) for Re $=10^{4} \mathrm{vs}$. the spanwise wavenumber $\alpha$, as calculated with the exact model (solid lines) and with the reduced model through Eq. (47) (dashed lines), in the case $x_{\text {in }}=10^{-5}$ for the indicated values of $\beta$. Right: as in left plot, but when $x_{\text {in }}=0.25$. Results by Tumin ${ }^{11}$ for $\beta=0.353$ and $x_{\text {in }}=0.25$ are plotted with plain circles. 
Summarizing, the reduced model (30)-(32) provides good results in both reconstructing the streamwise evolution of the streaks (including the MUSM and transient evolutions) and computing the optimal perturbations.

\section{CONCLUSIONS}

The low dimensional structure of streak perturbations in a wedge flow boundary layer (which includes the Blasius boundary layer as a particular case) has been analyzed relying on an approximate self similar behavior of the streaks. The starting point of this analysis was the well known property that the wall-normal profiles of the streamwise velocity component, $\hat{U}$, remains proportional to itself along the streamwise coordinate. In other words, such profile can be approximately described by just one mode. The remaining velocity components do not show this property and thus reducing the description of streaks to the integration of a few ODEs is not obvious. Application of proper orthogonal decomposition to a set of snapshots providing the wall normal profiles of the velocity components showed that five modes would be necessary to obtain a reasonable approximation. And furthermore, application of standard POD-based model reduction would not provide physical insight in the low dimensional structure of the process. Thus, a more ad hoc procedure has been followed in this paper that both is based physically meaningful modes and aims a more drastic reduction of the relevant degrees of freedom. As a previous step to derive the low dimensional model, two additional quantities involving the cross flow velocities, $\hat{H}=\hat{V}+\hat{W}$ and $\hat{V}^{\prime}=\partial_{\zeta} \hat{V}+g(\hat{x}) \hat{V}$, were identified in Subsection III A that were suggested by the asymptotic behavior of the streaks near the free stream and showed a one-mode structure. Projection of the governing equations onto the modes associated with $\hat{U}, \hat{H}$, and $\hat{V}^{\prime}$, produced a reduced model consisting in three ODEs (Eqs. (30)-(32)), which can be seen as one-dimensional analogs of the continuity, the streamwise momentum, and the streamwise vorticity equations. This system of equations has a first integral (Eq. (38)), which implied that the reduced model exhibits only two degrees of freedom. Integration of this system produces results that compare quite well with the exact counterparts in connection with:

- The (algebraic) asymptotic behavior near the leading edge, since the most dangerous exponent is well approximated (see Fig. 3).

- The streamwise evolution of the wall normal maxima of the velocity components (see Fig. 4), including both the MUSM and the transient streamwise evolution approaching the MUSM.

- The reconstruction of the wall normal profiles of the velocity components, including the cross flow components $\hat{V}$ and $\hat{W}$ (see Fig. 5), which do not show a one-mode behavior.

- The calculation of optimal streaks (see Fig. 6).

The low dimensional model derived in this paper may be regarded as a first step to derive simpler as well as reliable low dimensional models (ODEs) to describe the essence of the interaction of streaks with mono-chromatic Tollmien-Schlichting/Squire waves, which is currently under research. This interaction is relevant to obtain simplified descriptions of some recent ideas on using streaks to delay transition. ${ }^{30}$ In addition, this paper contains non-standard ideas to get low dimensional models in presence of approximate symmetries, which are frequent in fluid mechanics (when some numerically obtained coefficients, which are formally of order one, are numerically small). Thus, we expect that the results in this paper be a step further in current efforts to obtain simplified, yet reasonably precise descriptions of some of the various transition mechanisms.

\section{ACKNOWLEDGMENTS}

This research was partially supported by the Spanish Ministry of Education (Grant No. TRA2010-18054). We are also indebted to an anonymous referee for some useful suggestions on an earlier version of the manuscript. 


\section{APPENDIX: COEFFICIENTS OF THE EQUATIONS INVOLVED IN THE LOW DIMENSIONAL MODEL}

The following mathematical expressions of the coefficients of the system of Eqs. (30)-(32) are given in terms of the $L_{2}$-inner product of the indicated functions:

$$
\begin{aligned}
& \gamma_{1}=2 \frac{\left\langle\tilde{V}_{1}^{\prime}, \zeta \tilde{U}_{1}^{\prime}\right\rangle_{L_{2}}}{\left\langle\tilde{V}_{1}^{\prime}, \tilde{U}_{1}\right\rangle_{L_{2}}}, \quad \gamma_{2}=-\frac{\left\langle\tilde{V}_{1}^{\prime}, \tilde{V}_{1}^{\prime}\right\rangle_{L_{2}}}{\left\langle\tilde{V}_{1}^{\prime}, \tilde{U}_{1}\right\rangle_{L_{2}}}, \quad \gamma_{3}=\frac{\left\langle V_{1}^{\prime}, \tilde{H}_{1}\right\rangle_{L_{2}}}{\left\langle\tilde{V}_{1}^{\prime}, \tilde{U}_{1}\right\rangle_{L_{2}}}, \\
& \gamma_{4}=-\lambda_{1}-\frac{\left\langle\tilde{U}_{1}, F^{\prime \prime} \tilde{V}_{1}\right\rangle_{L_{2}}}{\left\langle\tilde{U}_{1}, F^{\prime} \tilde{U}_{1}\right\rangle_{L_{2}}}+\frac{\left\langle\tilde{U}_{1}, \tilde{U}_{1}\right\rangle_{L_{2}}}{\left\langle\tilde{U}_{1}, F^{\prime} \tilde{U}_{1}\right\rangle_{L_{2}}} g(\hat{x})^{2}, \\
& \gamma_{5}=\frac{(\beta-1)\left\langle\tilde{H}_{1}^{\prime},\left[F+(2 \beta-1) \zeta F^{\prime}+(\beta-1) \zeta^{2} F^{\prime \prime}\right] \tilde{U}_{1}\right\rangle_{L_{2}}}{(2-\beta)^{2}\left\langle\tilde{H}_{1}^{\prime},\left(F^{\prime} \tilde{H}_{1}\right)^{\prime}\right\rangle_{L_{2}}} g(\hat{x}), \\
& \gamma_{6}=\frac{1-\beta}{2-\beta}-\lambda_{1}+\frac{\left\langle\tilde{H}_{1}^{\prime}, \tilde{H}_{1}^{\prime}\right\rangle_{L_{2}}}{\left\langle\tilde{H}_{1}^{\prime},\left(F^{\prime} \tilde{H}_{1}\right)^{\prime}\right\rangle_{L_{2}}} g(\hat{x})^{2}, \\
& \Psi_{1}=-\frac{\left\langle\tilde{H}_{1}^{\prime}, F^{\prime} \tilde{V}_{1}^{\prime}\right\rangle_{L_{2}}}{\left\langle\tilde{H}_{1}^{\prime},\left(F^{\prime} \tilde{H}_{1}\right)^{\prime}\right\rangle_{L_{2}}}-\frac{\left\langle\tilde{H}_{1}, F^{\prime \prime} K(\hat{x}, \zeta)\right\rangle_{L_{2}}}{\left\langle\tilde{H}_{1}^{\prime},\left(F^{\prime} \tilde{H}_{1}\right)^{\prime}\right\rangle_{L_{2}}}, \quad \Psi_{2}=-\frac{\left\langle\tilde{U}_{1}, F^{\prime \prime} K(\hat{x}, \zeta)\right\rangle_{L_{2}}}{\left\langle\tilde{U}_{1}, F^{\prime} \tilde{U}_{1}\right\rangle_{L_{2}}}, \\
& \Psi_{3}=\frac{\left\langle\tilde{H}_{1}^{\prime}, F \tilde{V}_{1}^{\prime \prime}+2 F^{\prime} \tilde{V}_{1}\right\rangle_{L_{2}}}{(2-\beta)\left\langle\tilde{H}_{1}^{\prime},\left(F^{\prime} \tilde{H}_{1}\right)^{\prime}\right\rangle_{L_{2}}}+ \\
& \frac{\left\langle\tilde{H}_{1}^{\prime}, \tilde{V}_{1}^{\prime \prime \prime}+F^{\prime \prime}[1+(\beta-1) g(\hat{x}) \zeta] K(\hat{x}, \zeta)-(2-\beta) \tilde{V}_{1} g(\hat{x})^{2}\right\rangle_{L_{2}}}{(2-\beta)\left\langle\tilde{H}_{1}^{\prime},\left(F^{\prime} \tilde{H}_{1}\right)^{\prime}\right\rangle_{L_{2}}},
\end{aligned}
$$

where $\lambda_{1}$ is the largest eigenvalue associated to the eigenfunction $\left(\tilde{U}_{1}, \tilde{V}_{1}, \tilde{H}_{1}\right)$, solution of the eigenvalue problem defined in Eqs. (19)-(22) and that we have called along the paper first Tumin mode.

${ }^{1}$ Y. S. Kachanov, "Physical mechanisms of laminar-boundary-layer transition," Annu. Rev. Fluid Mech. 26, 411 (1994).

${ }^{2}$ P. S. Klebanoff, "Effect of free-stream turbulence on a laminar boundary layer," Bull. Amer. Phys. Soc. 10(11), 1323 (1971).

${ }^{3}$ L. S. Hultgren and L. H. Gustavsson, "Algebraic growth of disturbances in a laminar boundary layer," Phys. Fluids 24, 1000 (1981).

${ }^{4}$ P. J. Schmid, "Nonmodal stability theory,” Annu. Rev. Fluid Mech. 39, 129 (2007).

${ }^{5}$ M. T. Landahl, "A note on the algebraic instability of inviscid paralell shear flows," J. Fluid Mech. 98, 243 (1980).

${ }^{6}$ P. Luchini, "Reynolds-number-independent instability of boundary layer over a flat surface," J. Fluid Mech. 327, 101 (1996).

${ }^{7}$ A. Tumin, "A model of spatial algebraic growth in a boundary layer subjected to a streamwise pressure gradient," Phys. Fluids 13, 1521 (2001).

${ }^{8}$ K. M. Butler and B. F. Farrel, "Three-dimensional optimal perturbations in viscous shear flow," Phys. Fluid A 4(8), 1637 (1992).

${ }^{9}$ P. Andersson, M. Berggren, and D. S. Henningson, "Optimal disturbances and bypass transition in boundary layers," Phys. Fluids 11, 134 (1999).

${ }^{10}$ P. Luchini, "Reynolds-number-independent instability of boundary layer over a flat surface: optimal perturbations," J. Fluid Mech. 404, 289 (2000).

${ }^{11}$ A. Tumin and D. E. Ashpis, "Optimal disturbances in boundary layers subject to streamwise pressure gradient," AIAA J. 41, 2297 (2003).

${ }^{12}$ K. J. A. Westin, A. V. Boiko, B. G. B. Klingmann, V. V. Kozlov, and P. H. Alfredsson, "Experiments in a boundary layer subjected to free stream turbulence. Part 1. boundary structure and receptivity," J. Fluid Mech. 281, 193 (1994).

${ }^{13}$ M. Higuera and J. M. Vega, "Modal description of internal optimal streaks," J. Fluid Mech. 626, 21 (2009).

${ }^{14}$ J. J. Sanchez-Alvarez, M. Higuera, and J. M. Vega, “Optimal streaks in a Falkner-Skan boundary layer,” Phys. Fluids 23, 024104-1 (2011).

15 J. L. Lumley, “Coherent structures in turbulence," in Transition and Turbulence, ed. R. E. Meyer, pp. 215-242 (1971).

${ }^{16}$ N. Aubry, P. Holmes, J. L. Lumley, and E. Stone, "The dynamics of coherent structures in the wall region of a turbulent boundary layer," J. Fluid Mech. 192, 115 (1988). 
${ }^{17}$ A. E. Deane, I. G. Kevrekidis, G. A. Karniadakis, and S. A. Orzag, "Low dimensional models for complex geometry flows: Application to grooved channels and circular cylinders," Phys. Fluids 3(10), 2337 (1991).

${ }^{18}$ J. Moehlis, T. R. Smith, P. Holmes, and H. Faisst, "Models for turbulent plane Couette flow using the proper orthogonal decomposition," Phys. Fluids 14, 2493 (2002).

${ }^{19}$ T. R. Smith, J. Moehlis, and P. Holmes, "Low-dimensional models for turbulent plane Couette flow in a minimal flow unit," J. Fluid Mech. 538, 71 (2005).

${ }^{20}$ F. Waleffe, "On a self-sustaining process in shear flows," Phys. Fluids 9, 883 (1997).

${ }^{21}$ L. H. O. Hellström, A. Sinha, and A. J. Smits, "Visualizing the very-large-scale motions in turbulent pipe flow," Phys. Fluid 23, 011703 (2011).

${ }^{22}$ V. M. Falkner and S. W. Skan, "Solutions of the boundary-layer equations," Phil. Mag. 12, 865 (1931).

${ }^{23}$ K. K. Chen and P. A. Libby, "Boundary layers with small departures from the Falkner-Skan profile," J. Fluid Mech. 33, 273 (1968)

${ }^{24}$ M. Couplet, C. Basdevant, and P. Sagaut, "Calibrated reduced-order POD-Galerkin system for fluid flow modelling," J. Comp. Phys. 207, 192 (2005).

${ }^{25}$ V. Kalb and A. Deane, "An intrinsic stabilization scheme for proper orthogonal decomposition based on low dimensional models," Phys. Fluids 19, 054106 (2007).

${ }^{26}$ M. L. Rapun and J. M. Vega, "Reduced order models based on local POD plus Galerkin projection," J. Comput. Phys. 229, 3046 (2010).

${ }^{27}$ S. Sirisup, G. A. Karniadakis, and I. G. Kevrekidis, "Equation free/Galerkin free POD assisted computation of incompressible flows," J. Comput. Phys. 207, 568 (2005).

${ }^{28}$ S. Sirisup and G. Karniadakis, "Stability and accuracy of periodic flow solutions obtained by a POD-penalty method," Physica D 202, 218 (2005).

${ }^{29}$ F. Terragni and J. M. Vega, "Local POD plus Galerkin projection in the unsteady lid-driven cavity problem," SIAM J. Sci. Comput. (USA) 33, 3538 (2011).

${ }^{30}$ P. Schlatter, E. Deusebio, H. C. de Lange, and L. Brandt, "Numerical study of the stabilisation of boundary-layer disturbances by finite amplitude streaks," J. Flow Control 2(4), 259 (2010). 
Physics of Fluids is copyrighted by the American Institute of Physics (AIP). Redistribution of journal material is subject to the AIP online journal license and/or AIP copyright. For more information, see http://ojps.aip.org/phf/phfcr.jsp 